“ (C) 2017 IEEE. Personal use of this material is permitted. Permission from IEEE must be obtained for all other uses, in any current or future media, including

reprinting/republishing this material for advertising or promotional purposes, creating new collective works, for resale or redistribution to servers or lists, or reuse of any copyrighted component of this work in other works." 


\title{
Overcoming Traditional Electrically Small Antenna Tradeoffs with Meta-Structures
}

\author{
Richard W. Ziolkowski ${ }^{1,2} *$ and Ming-Chun Tang ${ }^{3}$ \\ ${ }^{1}$ University of Technology Sydney, Global Big Data Technologies Centre, Ultimo NSW 2000, Australia \\ ${ }^{2}$ Department of Electrical and Computer Engineering, University of Arizona, Tucson, AZ, USA, ziolkowski@ece.arizona.edu \\ ${ }^{3}$ College of Communication Engineering, Chongqing University, Chongqing, 400044, China, tangmingchun@cqu.edu.cn
}

\begin{abstract}
Metamaterial-inspired near-field resonant parasitic (NFRP) electrically small antennas (ESAs) have been designed and experimentally validated to have not only high radiation efficiencies, but also multi-functionality, large bandwidths, high directivities and reconfigurability. These expanded capabilities have been attained by introducing more complex metastructures, i.e., multiple NFRP elements loaded with fixed and tunable lumped elements, as well as active circuits. Different classes of passive and active NFRP ESAs that have successfully produced these effects will be reviewed, and several recently reported ESA systems will be introduced and discussed.
\end{abstract}

Index Terms-Bandwidth, directivity, electrically small antennas, frequency agility, Huygens source, parasitic elements

\section{INTRODUCTION}

Mobile platform technologies continue to evolve with an ever increasing desire for compactness and more functionality. This trend has generated an intense demand for printed electrically small antennas (ESAs) with superior performance characteristics. It will be even more intense with the growth of IoT (internet-of-things) applications.

There have been many approaches reported recently to meet these increasing demands. Many have been associated with metamaterials [1]-[4]. We have emphasized metamaterialinspired structures to achieve many different classes of ESAs [5]. In particular, by incorporating near-field resonant parasitic (NFRP) elements designed and tuned specifically for particular driven radiating elements, nearly complete matching to the source and highly efficient systems have been realized. By loading the NFRP elements with lumped element components and circuits rather than the driven element, exquisite control of the difficult tradeoff factors, e.g., bandwidth, beam width, directivity, and front-to-back ratio (FTBR), has been achieved. In particular, by introducing several NFRP elements and circuit-augmented versions, we have realized multi-functional, tunable, reconfigurable, low-profile, and high directivity ESAs. Many of these designs will be discussed in our presentation. Only a few will be highlighted in this article.

\section{MULTIFUnCTIONAL ESAS}

Consider the frequency agile circularly polarized (CP) antenna shown in Fig. 1. It is derived from the single (GPS L1) and dual frequency (GPS L1 and L2) CP antennas reported in [7], which incorporated, respectively, two and four NFRP elements. Those resonators were tuned to achieve the $\mathrm{CP}$ behavior without needing any phase shifter(s), and these antenna systems required only one source. By adding varactors into the protractor, i.e., capacitively loaded loop (CLL), NFRP elements, a frequency agile $\mathrm{CP}$ antenna has been achieved. The fabricated antenna is shown in Fig. 2.

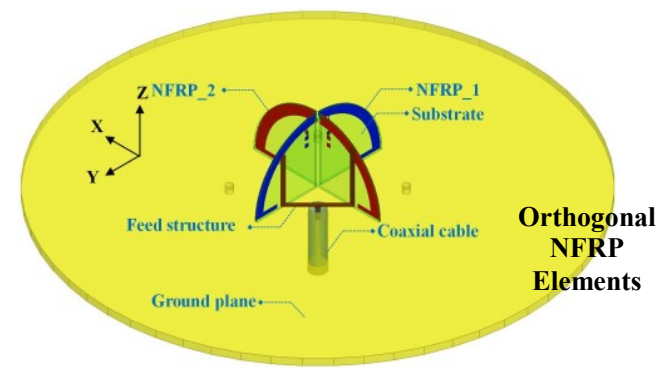

Fig. 1 Isometric view of the varactor-enabled, frequency-agile CP NFRP ESA [6].

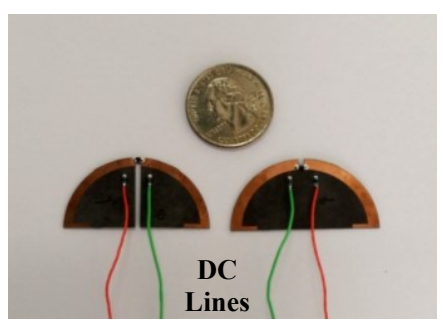

(a)

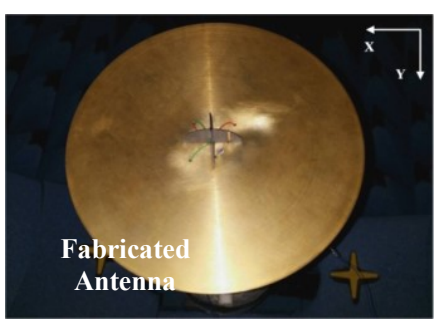

(b)
Fig. 2 Fabricated frequency-agile CP NFRP ESA. (a) Two NFRP elements before integration with the ground disc, and (b) 3-D isometric view of the fabricated antenna in the measurement chamber. [6]

The measured prototype ESA exhibited a wide 3-dB AR frequency agility range, $53.7 \mathrm{MHz}(\approx 3.92 \%$ fractional bandwidth from 1.3416 to $1.3956 \mathrm{GHz}$ ). This outcome was more than four times larger than the original fixed capacitor $(5.0 \mathrm{pF})$ version of this antenna, which had only a $0.84 \%$ fractional CP bandwidth. Moreover, the antenna was found to have a good impedance match, reasonably high radiation efficiency, and stable and uniform CP radiation patterns over its entire frequency-agile range [6].

In another multi-functional design, we have incorporated filters into the antenna. Filtennas are codesigned antennas that combine a radiating element and a filter into a single module. 
Because of its self-contained filtering function, a filtenna has several advantages over common antennas when a signal is received. It reduces the interconnection losses that occur when a standard receiving antenna is connected to a filter in the processing layer. It suppresses unwanted signals out of the operational band. Moreover, from a practical point of view, it facilitates a more compact and lower cost RF front-end system.

We have reported two electrically small, efficient, planar, monopole filtennas based on capacitively loaded loop (CLL) resonators [8]. The CLL NFRP elements provided the filtering characteristics. A drawing of one of these antennas and a photo of its experimentally validated prototype are shown in Fig. 3. The experimental results demonstrated that this electrically small system had a $145 \mathrm{MHz}$ impedance bandwidth (from 2.24 to $2.385 \mathrm{GH}$, a $6.27 \%$ fractional bandwidth), high out-of-band rejection, and stable omnidirectional radiation patterns.

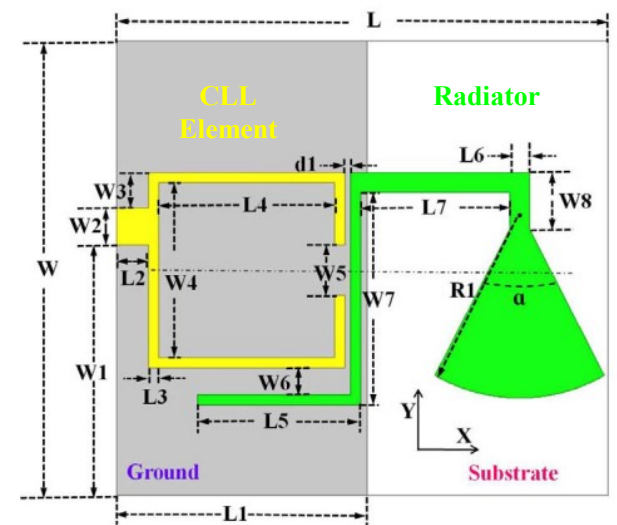

(a)

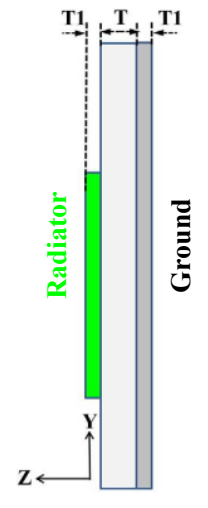

(b)

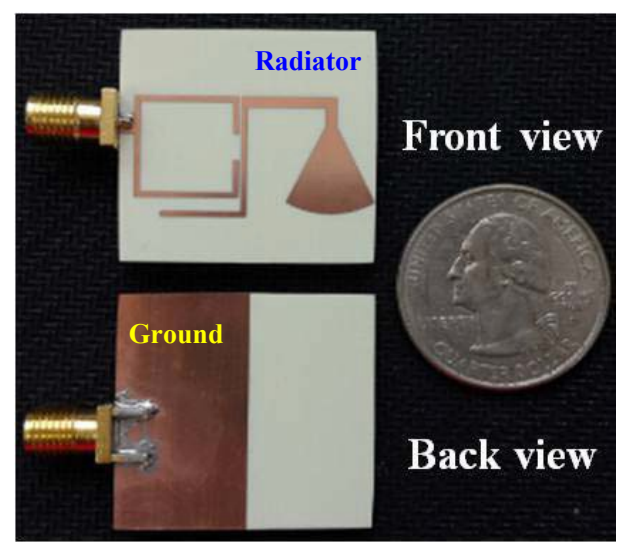

(c)

Fig. 3. Electrically small NFRP filtenna. (a) Top view and (b) side view of the HFSS model. (c) Front and back views of the fabricated prototype [8].

\section{ENDFIRE NFRP ESAS}

By combining two or more elements together in an end-fire configuration, one being driven and the other being a NFRP element, it has been demonstrated that significant directivity enhancements can be realized in flexible ESA designs [9], [10]. An example of this approach is the compact two element array shown in Fig. 4 [10]. It is a flexible printed NFRP GPS L1 ESA that is an efficient and directive radiator.
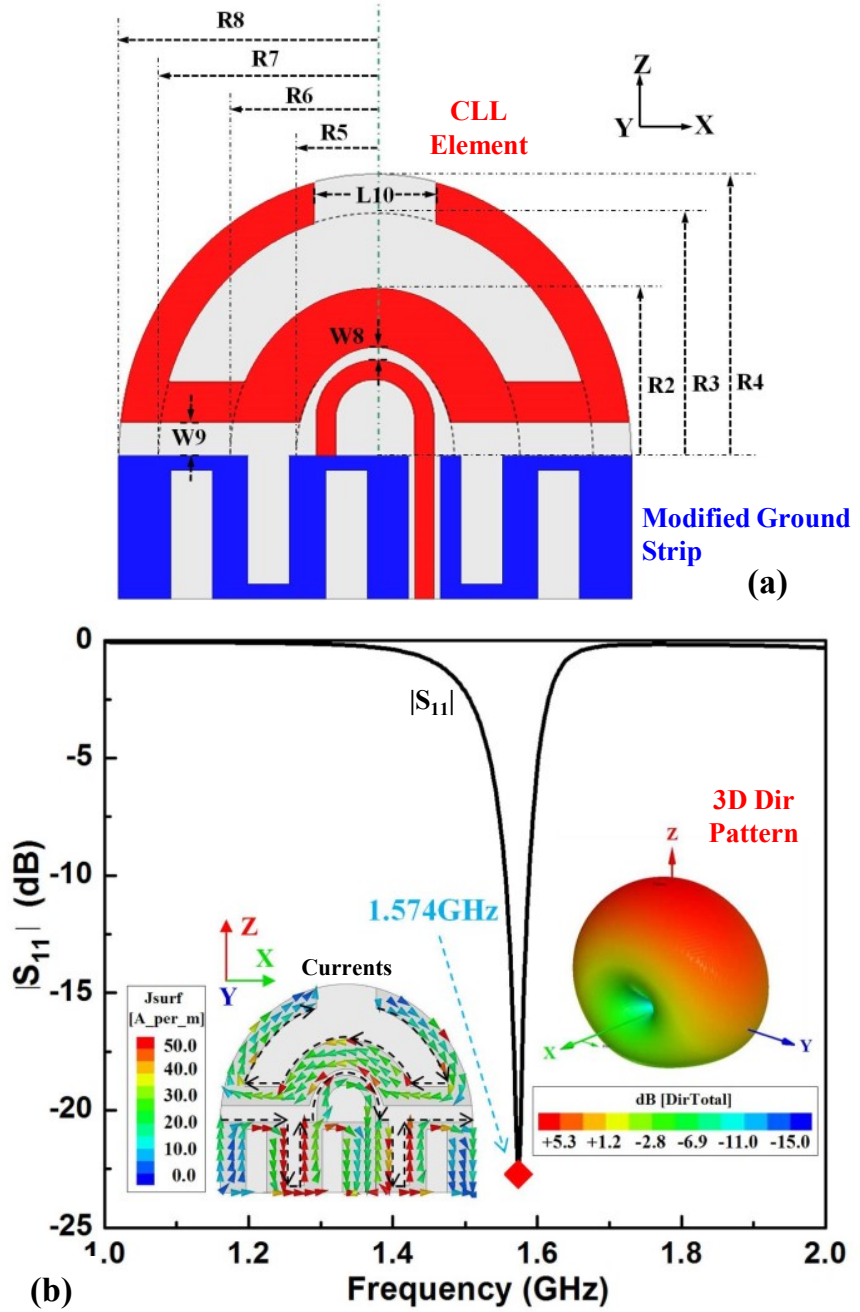

Fig. 4. Printed, flexible, modified CPW-feedline, electrically small antenna operates as an end-fire, two element dipole array. Both the modified CPWfeedline and the CLL NFRP element act as electric dipole radiators. (a) Basic design, and (b) $\left|S_{11}\right|$ values as functions of the excitation frequency. The inset figures represent the current distributions on the metal components and the $3 \mathrm{D}$ directivity pattern. The latter illustrates the enhanced directivity that occurs along the positive $\mathrm{z}$-axis away from the source [10].

The simple uniplanar design incorporates a capacitively loaded loop (CLL) resonator, which acts as the NFRP element, and a coplanar waveguide (CPW)-fed semi-loop antenna as the driven element. A set of slots are introduced into the CPW feedline ground strips. The resulting meanderline-shaped CPW ground strips act as a director dipole element that is capacitively coupled to the CLL NFRP element in such a manner that when they are properly tuned, nearly complete 
matching to the $50 \Omega$ source is achieved with no matching circuit, and the pair acts as a two-element electric dipole endfire array.

Parameter studies reported in [10] illustrate the nuances of the design and its operating mechanisms. The end-fire realized gain is $3.57 \mathrm{dBi}$ with the FTBR $=13.44 \mathrm{~dB}$ at its resonance frequency: $1.574 \mathrm{GHz}$ (GPS L1), where the electrical size $\mathrm{ka}=$ 0.97 . The flexibility of the proposed antenna was demonstrated both numerically and experimentally by mounting it on several cylindrical structures whose curvatures vary over a large range and by confirming that there is little impact on its operational frequency, impedance matching, bandwidth and radiation characteristics [10]. Fig. 5 illustrates the prototype in the testing environment in both bending configurations.

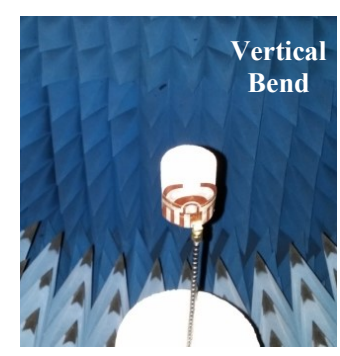

(a)

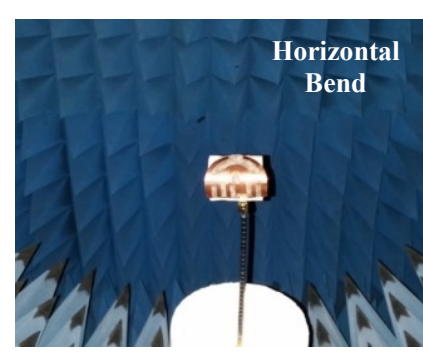

(b)
Fig. 5 The flexible, printed uniplanar, quasi-Yagi-Uda CPW-fed prototype antenna mounted on a $20 \mathrm{~mm}$ radius foam cylinder in the anechoic chamber was tested in two bending conditions. (a) Vertical bend, and (b) horizontal bend [10].

\section{BROADSIDE RADIATING HUYGENS SOURCE ESAS}

Huygens equivalence principle provides yet another means to achieve higher directivity. By combining together an electric dipole and a magnetic dipole in a plane, one can obtain a Huygens source, i.e., an antenna that radiates primarily into one of the half spaces bounded by that plane. The key design issues are to achieve the same phase center for both radiating elements and to have them both radiate equivalent amounts of power. Additional tuning is necessary to direct the radiated power into the desired half space.

Two recently reported broadside radiating, Huygens source ESA designs are shown in Fig. 6 [11], [12]. Both utilize a coax-fed printed dipole element to excite simultaneously Egyptian axe dipole (EAD) and CLL NFRP elements to obtain the Huygens source behavior. The EAD meta-structures produce the electric dipole behavior while the CLL metastructures produce the orthogonal magnetic dipole behavior. The $300 \mathrm{MHz}$ design illustrated in Fig. $6 \mathrm{a}$ is only $\sim \lambda / 80$ thick; its driven dipole is strongly coupled to the EAD NFRP element [11]. The height of the EAD meta-structure facilitates the needed phase centering, as well as controls the main emission direction along the broadside direction (either up or down along the z-axis). The $1.5 \mathrm{GHz}$ design shown in Fig. $6 \mathrm{~b}$ was fabricated and experimentally validated [12]. In this design, the CLL element is directly coupled to the driven dipole element and the EAD meta-structure consists of two mirrored pieces adjacent on both sides of the CLL element. Again, the height of the EAD meta-structure facilitates phase centering and controls the emission direction.

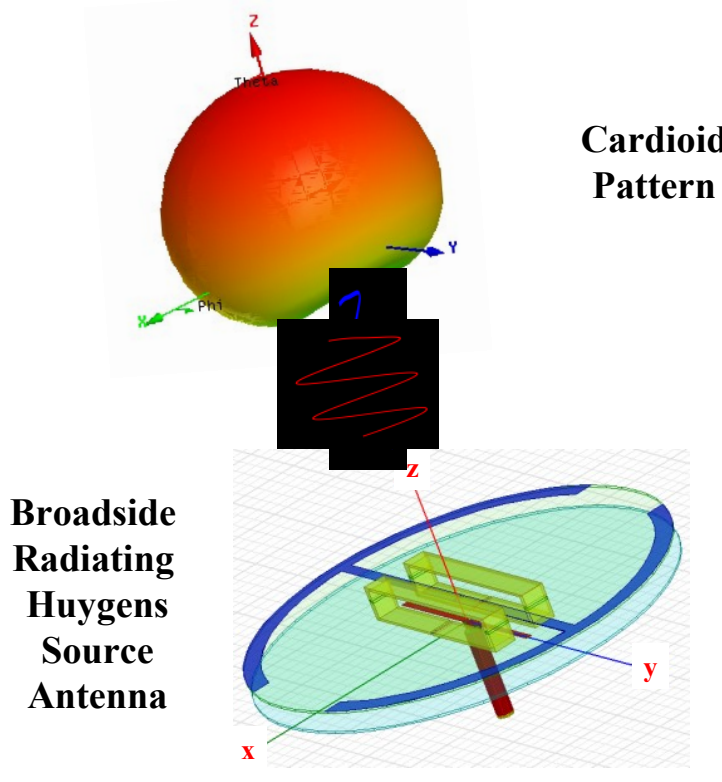

(a)

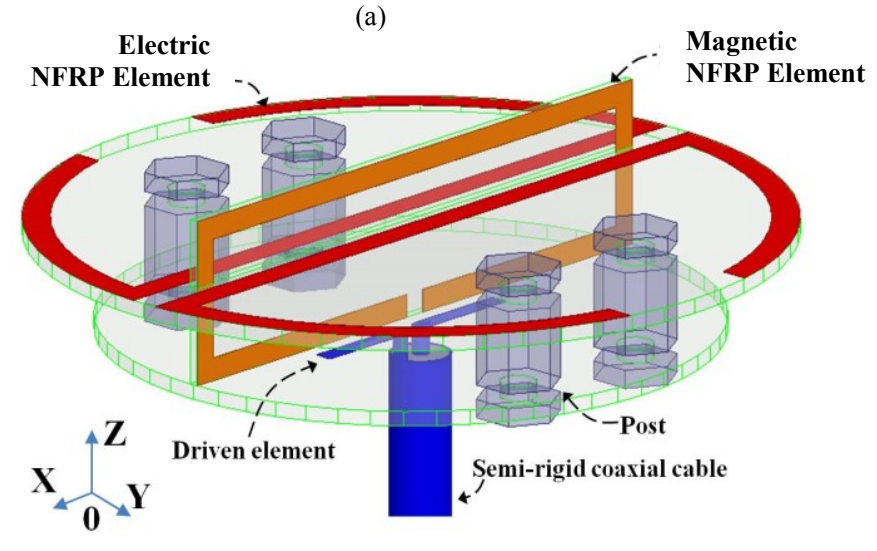

(b)

Fig. 6 Low profile, broadside radiating NFRP Huygens source ESAs. (a) Ideal case $(\sim \lambda / 80$ thick) [11], and (b) experimentally validated case $(\sim \lambda / 20$ thick) [12].

The $\left|\mathrm{S}_{11}\right|$ values as a function of the excitation frequency of the antenna were measured; the measured results together with the corresponding simulation values are shown in Fig. 7. In order to emphasize the contribution of the balun on the antenna performance, the simulated $\left|S_{11}\right|$ values when the antenna is attached to a shorter coax feedline $(3 \mathrm{~mm})$, and when it is attached to a longer coax feedline $(60 \mathrm{~mm})$ were obtained and are also plotted. The simulation (measurement) results were $\left|\mathrm{S}_{11}\right|_{\min }=-17.69 \mathrm{~dB}(-16.38 \mathrm{~dB})$ at $f_{0}=1.502 \mathrm{GHz}$ $(1.496 \mathrm{GHz})$ and the corresponding $-10 \mathrm{~dB}$ impedance bandwidth was $8.1 \mathrm{MHz}(9.3 \mathrm{MHz})$. Thus, the total height and electrical size at $f_{0}$ of the measured (simulated) Huygens 
source antenna system were, respectively, $\lambda_{0} / 20.37=0.0491$ $\lambda_{0}\left(\lambda_{0} / 20.45=0.0489 \lambda_{0}\right)$ and $k a=0.645(0.642)$. These values confirmed that the prototype Huygens source antenna was low-profile and electrically small. Moreover, the measured $-10-\mathrm{dB}$ impedance bandwidth was more than 10 times larger than that reported for the idealized version shown in Fig. 6a [11].

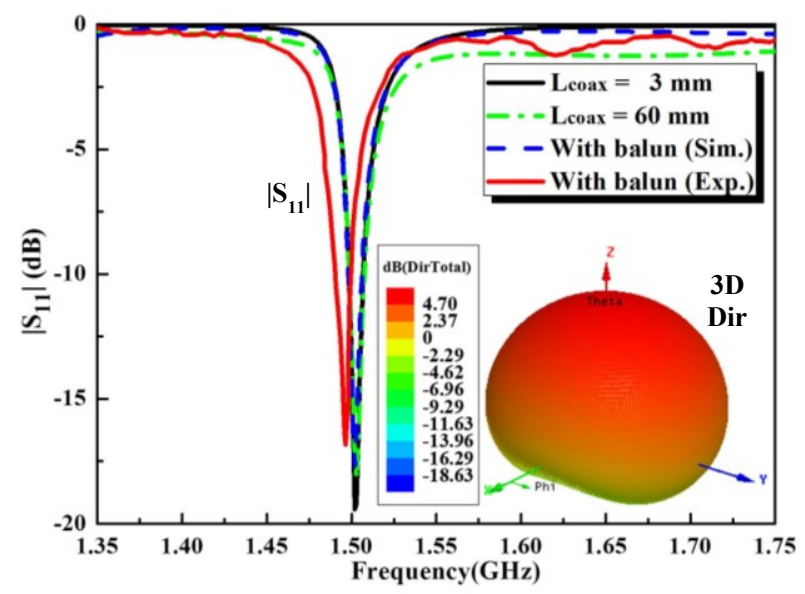

Fig. 7. Simulated and measured $\left|\mathrm{S}_{11}\right|$ values versus the excitation frequency for the forward radiating Huygens source antenna, together with the simulated $3 \mathrm{D}$ directivity pattern at the resonance frequency, $1.502 \mathrm{GHz}$, for the $3.0 \mathrm{~mm}$ coaxial feedline case [12].

\section{CONCLUSION}

Several combinations of metamaterial-inspired electrically small NFRP elements, and driven electrically small radiators were reviewed. The proficiency to design electrically small antenna systems with combinations of these meta-structures to achieve multi-functionality and higher directivity that can be achieved with any single electrically small antenna was illustrated. These and other related experimentally validated NFRP ESAs will be emphasized in our presentation.

\section{References}

[1] G. V. Eleftheriades, M. A. Antoniades, and F. Qureshi, "Antenna applications of negative-refractive-index transmission-line structures," IET microwaves, antennas \& propagation, vol. 1, no. 1, Feb. 2007.

[2] C. Caloz, T. Itoh, and A. Rennings, "CRLH metamaterial leaky-wave and resonant antennas," IEEE Antennas Propag. Mag., vol. 50, no. 5, pp. 25-39, Oct. 2008.

[3] M. A. Antoniades, J. Zhu, M. Selvanayagam, and G. V. Eleftheriades, "Compact, wideband and multiband antennas based on metamaterial concepts," in Proc. of the Fourth European Conference on Antennas and Propagation, IEEE, Barcelona, Spain, Apr. 2010, pp. 1-5.

[4] Y. Dong and T. Itoh, "Metamaterial-based antennas," Proc. IEEE, vol. 100, no. 7, pp. 2271-2285, Jul. 2012.

[5] R. W. Ziolkowski, P. Jin, and C.-C. Lin, "Metamaterial-inspired engineering of antennas," Proc. IEEE, vol.99, no.10,pp.1720-1731,Oct. 2011 .

[6] M.-C. Tang, and R. W. Ziolkowski, "Frequency-agile, efficient, circularly polarized, near-field resonant antenna: Designs and measurements," IEEE Trans. Antennas Propag., vol. 63, no. 11, pp. 5203-5209, Nov. 2015.

[7] P. Jin and R. W. Ziolkowski, "Multi-frequency, linear and circular polarized, metamaterial-inspired near-field resonant parasitic antennas," IEEE Trans. Antennas Propag., vol. 59, pp. 1446-1459, May 2011.

[8] M.-C. Tang, Y. Chen, and R. W. Ziolkowski, "Experimentally validated, planar, wideband, electrically small, monopole filtennas based on capacitively loaded loop resonators," IEEE Trans. Antennas Propag., vol. 64, no. 8, pp. 3353-3360, Aug. 2016.

[9] M.-C. Tang, T. Shi and R. W. Ziolkowski, "Flexible efficient quasi-Yagi printed uniplanar antenna," IEEE Trans. Antennas Propag., vol. 63, no. 12, pp. 5343-5350, Dec. 2015.

[10] M.-C. Tang, B. Zhou and R. W. Ziolkowski, "Flexible uniplanar electrically small directive antenna empowered by a modified CPWfeed," IEEE Antennas Wireless Propag. Lett., vol. 15, pp. 914-917, 2016.

[11] R. W. Ziolkowski, "Low profile, broadside radiating, electrically small Huygens source antennas," IEEE Access, vol. 3, pp. 2644-2651, Dec. 2015.

[12] M.-C. Tang, H. Wang, and R. W. Ziolkowski, "Design and testing of simple, electrically small, low-profile, Huygens source antennas with broadside radiation performance," to appear in IEEE Trans. Antennas Propag., 2016. 